\title{
Anti-CD19 CAR T cells with high-dose melphalan and autologous stem cell transplantation for refractory multiple myeloma
}

\author{
Alfred L. Garfall, ${ }^{1}$ Edward A. Stadtmauer, ${ }^{1}$ Wei-Ting Hwang, ${ }^{1}$ Simon F. Lacey, ${ }^{1}$ \\ Jan Joseph Melenhorst, ${ }^{1}$ Maria Krevvata, ${ }^{1}$ Martin P. Carroll, ${ }^{1}$ William H. Matsui, ${ }^{2}$ Qiuju Wang, ${ }^{2}$ \\ Madhav V. Dhodapkar, ${ }^{3}$ Kavita Dhodapkar, ${ }^{3}$ Rituparna Das, ${ }^{3}$ Dan T. Vogl, ${ }^{1}$ Brendan M. Weiss, \\ Adam D. Cohen, ${ }^{1}$ Patricia A. Mangan, ${ }^{1}$ Emily C. Ayers, ${ }^{1}$ Selene Nunez-Cruz,, ${ }^{1}$ rina Kulikovskaya, \\ Megan M. Davis, ${ }^{1}$ Anne Lamontagne, ${ }^{1}$ Karen Dengel, ${ }^{1}$ Naseem D.S. Kerr, ${ }^{1}$ Regina M. Young, \\ Donald L. Siegel, ${ }^{1}$ Bruce L. Levine, ${ }^{1}$ Michael C. Milone, ${ }^{1}$ Marcela V. Maus, ${ }^{4}$ and Carl H. June ${ }^{1}$ \\ 'Perelman School of Medicine at the University of Pennsylvania, Philadelphia, Pennsylvania, USA. ${ }^{2} J o h n s$ Hopkins \\ University School of Medicine, Baltimore, Maryland, USA. ${ }^{3}$ Yale University School of Medicine, New Haven, Connecticut, \\ USA. ${ }^{4}$ Massachusetts General Hospital, Harvard Medical School, Boston, Massachusetts, USA
}

Authorship note: ALG and EAS contributed equally to this work.

Conflict of interest: ALG has received research funding from Novartis and consulting fees from Medimmune, Novartis, and Kite Pharma. ADC has received consulting fees from GlaxoSmithKline, Celgene, Janssen, Takeda, Bristol-Myers Squibb, and research support from Bristol-Myers Squibb. BMW has received research funding from Janssen and Prothena and consulting fees from Novartis and Alnylam. MVM has received consulting fees from Novartis, Juno, and Kite Pharma. BLL, MCM, MVM, and CHJ are inventors on patents in the field of cell and gene therapy held by the University of Pennsylvania (patents are listed at the end of the supplemental material). WTH, SFL, JMM, SNC, IK, BLL, MCM, and $\mathrm{CH}$ have received research funding from Novartis. BLL has received personal fees from GE Healthcare and Brammer Bio that are unrelated to the submitted work and is a cofounder and holds equity in Tmunity Therapeutics. PAM has received consulting fees from Kite Pharma.

Competing interests of authors from the University of Pennsylvania are managed in accordance with University policies.

Submitted: February 12, 2018

Accepted: March 20, 2018

Published: $x x x x$

Reference information: JCI Insight. 2018;3(8):e120505 https://doi.org/10.1172/jci. insight.120505.
BACKCROUND. Multiple myeloma is usually fatal due to serial relapses that become progressively refractory to therapy. CD19 is typically absent on the dominant multiple myeloma cell population but may be present on minor subsets with unique myeloma-propagating properties. To target myeloma-propagating cells, we clinically evaluated autologous $T$ cells transduced with a chimeric antigen receptor (CAR) against CD19 (CTL019).

METHODS. Subjects received CTLO19 following salvage high-dose melphalan and autologous stem cell transplantation (ASCT). All subjects had relapsed/refractory multiple myeloma and had previously undergone ASCT with less than 1 year progression-free survival (PFS).

RESULTS. ASCT + CTL019 was safe and feasible, with most toxicity attributable to ASCT and no severe cytokine release syndrome. Two of 10 subjects exhibited significantly longer PFS after ASCT + CTL019 compared with prior ASCT (479 vs. 181 days; 249 vs. 127 days). Correlates of favorable clinical outcome included peak CTL019 frequency in bone marrow and emergence of humoral and cellular immune responses against the stem-cell antigen Sox2. Ex vivo treatment of primary myeloma samples with a combination of CTLO19 and CAR T cells against the plasma cell antigen BCMA reliably inhibited myeloma colony formation in vitro, whereas treatment with either CAR alone inhibited colony formation inconsistently.

CONCLUSION. CTL019 may improve duration of response to standard multiple myeloma therapies by targeting and precipitating secondary immune responses against myeloma-propagating cells.

TRIAL REGISTRATION. Clinicaltrials.gov identifier NCT02135406.

FUNDING. Novartis, NIH, Conquer Cancer Foundation.

\section{Introduction}

Multiple myeloma is a hematologic malignancy of bone marrow plasma cells, which are immunoglobulin-secreting B-lineage cells. Response rates and survival have improved for multiple myeloma patients with development of new therapies that target neoplastic plasma cells (1). Though most patients respond to modern first-line therapy, response durability remains inadequate. Nearly all patients, even those who achieve complete responses, eventually relapse and develop treatment-refractory, fatal disease.

Individual multiple myeloma cells may differ in their myeloma-propagating potential (i.e., their ability to give rise to clinical relapses) $(2,3)$. Specifically targeting cells with high myeloma-propagating capability would be expected to prevent or delay relapses. Heterogeneity within the myeloma clone for 


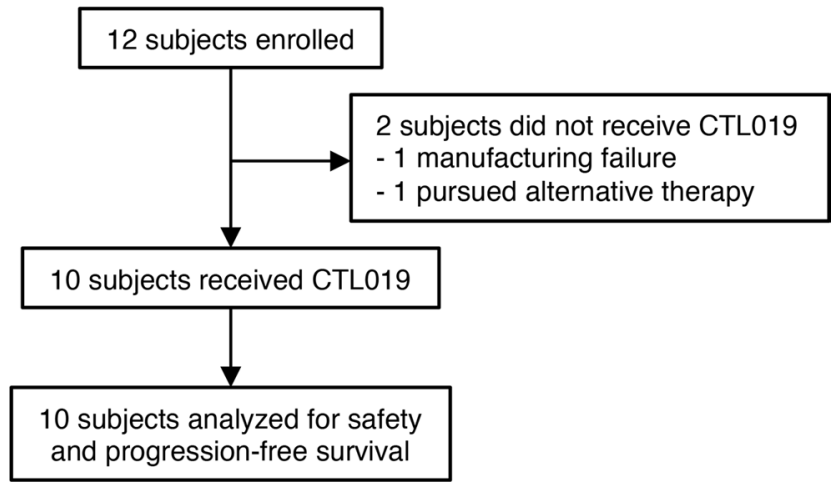

Figure 1. Subject flow diagram. myeloma-propagating capability may correspond to heterogeneity for cell-surface immunophenotype. Among multiple myeloma cells, the range of immunophenotypes recapitulates normal maturation of post-germinal-center B cells. The dominant population in most patients resembles the most differentiated normal plasma cell subset: $\mathrm{CD}^{+} 8^{+} \mathrm{CD} 138^{+} \mathrm{CD} 19^{-}$(4). Minor subsets of the multiple myeloma clone with less differentiated plasma cell phenotypes (CD138 ${ }^{10 /-}$, refs. $5-7$ or $\mathrm{CD} 19^{+}$, ref. 8$)$ or a B cell phenotype $\left(\mathrm{CD} 138^{-} \mathrm{CD} 19^{+} \mathrm{CD} 20^{+}\right)$can also be identified in patients (9-22). Studies comparing these immunophenotypic subsets for myeloma-propagating capability in vitro or in immunocompromised murine models have yielded conflicting results, variably identifying clonotypic B cells (23-27), CD138- plasma cells $(5,28)$, or the dominant CD138 ${ }^{+}$plasma cells $(22,29)$ as the most clonogenic subset. It therefore remains uncertain whether minor, less differentiated components of the multiple myeloma clone participate in disease pathogenesis. This is a clinically important question since many promising immunotherapies for multiple myeloma target single cell-surface antigens that may not be shared among all these subsets.

To address this question clinically, we conducted a pilot clinical trial of autologous $\mathrm{T}$ cells transduced with an anti-CD19 chimeric antigen receptor (CAR) (CTL019) in multiple myeloma patients. Since CD19 should be expressed on only a minority of myeloma cells, CTL019 alone would not be expected to yield clinical responses. We therefore combined CTL019 with high-dose melphalan and autologous stem cell transplantation (ASCT), a standard multiple myeloma therapy that we have previously used as a platform for delivery of autologous T cell therapies (30-34). To distinguish the clinical effects of ASCT from those of CTL019, we enrolled subjects who had previously undergone ASCT with poor response, defined as progression-free survival (PFS) of less than 1 year, and assessed whether ASCT + CTL019 resulted in longer PFS compared to each subject's prior ASCT without CTL019.

We previously reported the initial outcome of the first subject treated on this study (35). This subject entered a durable complete response after ASCT + CTL019, despite having attained only a 6-month partial response to first-line ASCT and having progressed through 9 subsequent lines of therapy. This outcome suggested clinical benefit from CTL019 despite absence of CD19 expression on the dominant neoplastic plasma cell population. Here, we report the remainder of the clinical and correlative results from this study.

\section{Results}

Patient characteristics. Twelve subjects enrolled in this clinical trial. Two subjects who enrolled did not receive study therapy due to failure of CTL019 manufacturing in one case and decision to pursue alternative therapy in the other. All remaining subjects received CTL019 and were analyzed for primary and secondary clinical endpoints including safety and PFS (Figure 1). Table 1 presents the clinical characteristics of the 10 subjects who received CTL019. No treated subjects were lost to follow-up. All subjects were treated according to the clinical trial protocol. Subjects were recruited and treated in 2014 and 2015. Median subject age was 61 (range 48-68) years. Most subjects' multiple myeloma exhibited genetic or clinical features associated with poor prognosis. Median total lines of therapy prior to ASCT + CTL019 was 6 (range 2-10). All subjects had previously received ASCT with a melphalan dose of $200 \mathrm{mg} / \mathrm{m}^{2}$ as a component of standard, first-line therapy. Melphalan dose for on-study ASCT was reduced to $140 \mathrm{mg} / \mathrm{m}^{2}$ from the standard 200 $\mathrm{mg} / \mathrm{m}^{2}$ dose in 7 of 10 subjects, as permitted by protocol, due to concern for excess toxicity in frail subjects or due to renal insufficiency.

CTL019 manufacturing feasibility and product characteristics. CTL019 manufacturing was attempted for 11 subjects. Supplemental Table 1 (supplemental material available online with this article; https://doi. org/10.1172/jci.insight.120505DS1) lists the CTL019 dose and transduction efficiency for each subject who received CTL019. In 10 of 11 subjects, the maximum planned dose of CTL019, $5 \times 10^{7}$ cells, was manufactured. In one subject, manufacturing was unsuccessful due to failure of autologous T cells to proliferate in culture. The median transduction efficiency was $10.1 \%$ (range 1.2-23.2), and the median total $\mathrm{T}$ cell dose was $4.4 \times 10^{8}$ (range $1.1 \times 10^{8}$ to $\left.6.0 \times 10^{8}\right)$.

Adverse events. Most adverse events were attributed to high-dose melphalan and were consistent with expectations in this patient population. Table 2 lists grade 3 or higher adverse events not related to disease progression 
Table 1. Patient characteristics and clinical responses

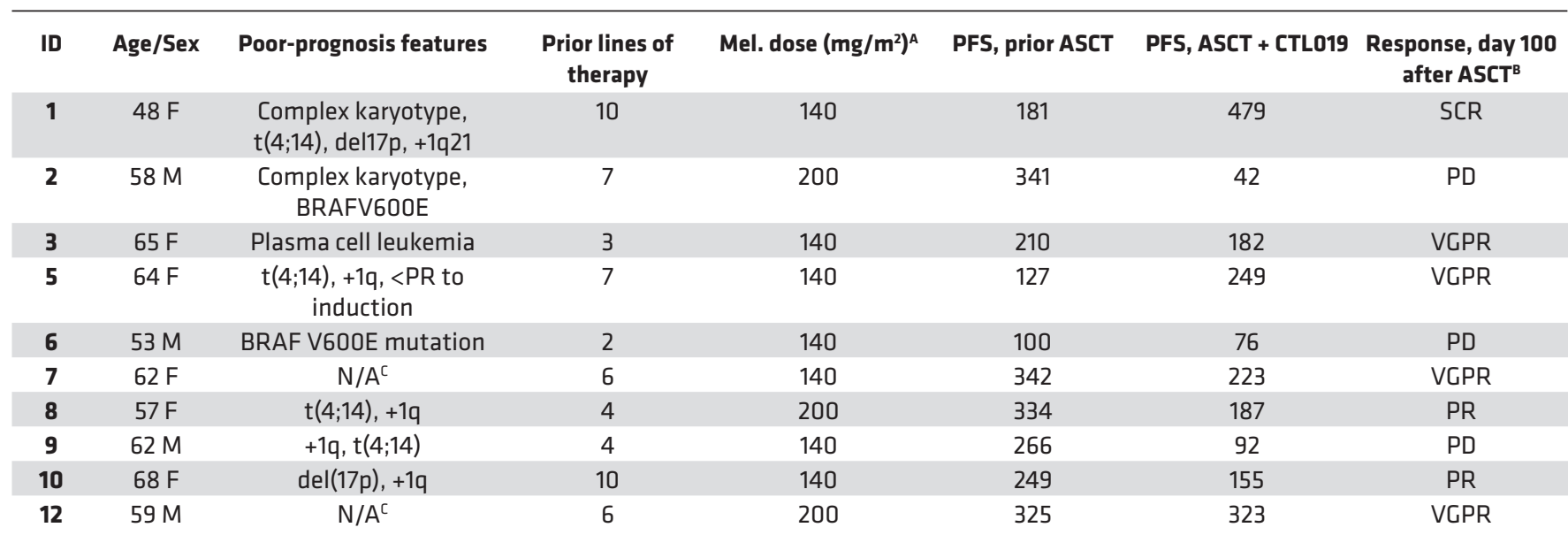

${ }^{A}$ Dose with ASCT + CTL019. All subjects received melphalan at $200 \mathrm{mg} / \mathrm{m}^{2}$ dose with prior ASCT. ${ }^{B}$ Responses are graded according to International Myeloma Working Group definitions. 'Cytogenetic data not available. Mel., melphalan; PFS, progression-free survival; VGPR, very good partial response; PR, partial response; $\mathrm{PD}$, progression of disease; SCR, stringent complete response.

that emerged after CTL019. Autologous gastrointestinal graft-versus-host disease (GVHD) occurred in one subject and was successfully treated with prednisone. Autologous GVHD is a known complication of ASCT (36), and the risk may be increased when ASCT is followed by infusion of activated autologous T cells (37). Cytokine release syndrome was observed only in one subject and was grade 1 in severity.

Response and PFS. At day 100 after ASCT, 8 of 10 subjects exhibited a partial response or better according to International Myeloma Working Group (IMWG) response criteria (38). This overall response rate is expected from ASCT alone, even in patients with advanced multiple myeloma (39). To assess whether CTL019 prolonged PFS after ASCT, we compared PFS after ASCT + CTL019 (PFS2) to each subject's PFS after prior ASCT (PFS1) (Table 1). In 8 of 10 subjects, PFS2 was shorter than PFS1. In subject 1 (as previously reported, ref. 35) and subject 5, however, PFS2 was substantially longer than PFS1 (479 vs. 181 days for subject $1 ; 249$ vs. 127 days for subject 5). Though both subjects 1 and 5 received low-dose maintenance therapy with lenalidomide after ASCT + CTL019, this could not explain the prolongation in PFS2 over PFS1; for subject 1, this maintenance regimen was less intense than the maintenance regimen administered after prior ASCT, and, for subject 5, maintenance therapy did not begin after ASCT + CTL019 until PFS2 had already exceeded PFS1. To assess how frequently PFS2 exceeds PFS1 generally in patients receiving second ASCT for advanced multiple myeloma, we conducted a retrospective analysis

Table 2. Grade 3 or higher adverse events emerging after CTL019

\begin{tabular}{|c|c|c|c|}
\hline Toxicity ${ }^{A}$ & Grade & $n$ & Relationship to CTL019 \\
\hline Thrombocytopenia & 4 & 1 & Unrelated \\
\hline Rash at catheter insertion site & 3 & 1 & Unrelated \\
\hline Pneumonia (parainfluenza) & 3 & 1 & Unrelated \\
\hline $\begin{array}{l}\text { Autologous graft-versus-host } \\
\text { disease (gastrointestinal) }\end{array}$ & 3 & 1 & Probably related \\
\hline Mucositis & 3 & 1 & Possibly related \\
\hline Infectious colitis (C. difficile) & 3 & 1 & Unrelated \\
\hline Hypotension & 3 & 1 & Unrelated \\
\hline
\end{tabular}

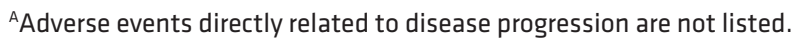


of 18 patients who received two ASCTs, first as a component of first-line therapy and second as salvage therapy for relapsed disease, at our institution between 2008 and 2015 and survived more than 30 days after transplant. In contrast to our ASCT + CTL019 cohort, where PFS2 exceeded PFS1 in 2 of 10 cases, none of the 18 salvage ASCT cases in the historical cohort exhibited PFS2 greater than PFS1 $(P=0.05)$ (Supplemental Table 2). We also examined the ratio of PFS2 to PFS1 in this historical cohort and the ASCT + CTL019 cohort. The PFS2/PFS1 ratio was significantly lower in the historical cohort than in the ASCT + CTL019 cohort (mean ratio 0.33 vs. 0.95 , median ratio 0.29 vs. $0.71 ; P=0.003$ ). Even excluding subjects 1 and 5, the outlier responders in the ASCT + CTL019 cohort, the PFS2/PFS1 ratio was still significantly more favorable in the ASCT + CTL019 cohort (mean ratio 0.33 vs. 0.62 , median ratio 0.29 vs. $0.64 ; P=$ 0.02). Though we recognize the limitations of comparisons to heterogeneous and small historical cohorts, these observations suggest that the substantially longer PFS2 compared with PFS1 in 2 of 10 subjects after ASCT + CTL019 is unlikely to have been due to second ASCT alone, and ASCT + CTL019 recipients generally exhibited longer PFS than expected based on historical expectations. These observations indicate potential clinical benefit from CTL019.

Clinical features at progression. Multiple myeloma can have myriad clinical manifestations including cytopenias, susceptibility to infection, bone destruction, hypercalcemia, impairment of renal function, and development of plasma cell tumors (plasmacytomas) that cause symptoms or organ dysfunction. At time of disease progression after ASCT + CTL019, the multiple myeloma in most subjects exhibited clinical features similar to each subject's prior instances of disease progression. In subjects 1 and 5, however, clinical features upon progression were distinct from the pre-CTL019 features of their multiple myeloma. Prior to ASCT + CTL019, both subjects 1 and 5 exhibited rapid disease rebound between therapies. In contrast, the rise in monoclonal immunoglobulin production at progression after ASCT + CTL019 was more gradual (Figure 2, A and B). At time of progression by serum monoclonal immunoglobulin criteria, bone marrow biopsies in subjects 1 and 5 showed no evidence of multiple myeloma by standard anatomic pathology assessment, and only very rare, $\mathrm{CD} 19^{-}$multiple myeloma plasma cells were detectable in bone marrow by flow cytometry (Figure 2, C-E), comprising $0.003 \%$ of cells in subject 1 and $0.006 \%$ of cells in subject 5 . In both subjects this contrasts with the heavy marrow infiltration that accompanied disease progression prior to ASCT + CTL019. Cross-sectional imaging showed multiple extramedullary plasmacytomas in both subjects as the only clinically significant disease manifestations (retroperitoneal and gluteal plasmacytomas in subject 1 , pleural plasmacytomas in subject 5 ). On positron emission tomography, the extramedullary plasmacytomas in both subjects 1 and 5 did not exhibit fluorodeoxyglucose uptake above background levels, suggesting indolent disease; this is unusual for extramedullary multiple myeloma, which is usually fluorodeoxyglucose-avid by this modality (40). Though multiple myeloma in subject 5 eventually developed a more aggressive and treatment-refractory clinical course, multiple myeloma of subject 1 remained indolent and uncharacteristically responsive to subsequent therapy. For example, serum IgA declined after radiation to one extramedullary plasmacytoma; the second plasmacytoma resolved, coinciding with normalization of serum multiple myeloma markers, after initiation of treatment with the anti-CD38 monoclonal antibody daratumumab (Figure 2A). Subject 1 remains without any clinical or serologic evidence of multiple myeloma more than 3.5 years after ASCT + CTL019 and more than 2 years after initial progression, despite having progressed through 10 lines of therapy during the 4 years prior to ASCT + CTL019. Collectively, these observations suggest that CTL019 converted the previously aggressive multiple myeloma in subjects 1 and 5 to a more indolent clinical behavior with disease growth confined, at least initially, to extramedullary sites despite the presence of rare multiple myeloma cells in bone marrow.

Baseline CD19 expression on multiple myeloma plasma cells. We analyzed CD19 expression on multiple myeloma plasma cells by flow cytometry on bone marrow aspirate obtained prior to ASCT (Supplemental Figure 1). Plasma cells were gated based on high expression of CD38 and then analyzed for expression of CD56, which is typically present on myeloma plasma cells but absent on normal plasma cells, and CD19. The dominant population of plasma cells was confirmed to be neoplastic based on monotypic expression of either kappa or lambda light chain (not shown) and, as expected, was CD19- in all evaluable cases. In 7 of 9 subjects with evaluable samples, minor $\mathrm{CD} 19^{+}$populations comprising $0.04 \%$ to $1.6 \%$ of plasma cells were identified (Supplemental Figure 1, left column). These CD19+ subpopulations exhibited monotypic kappa or lambda light chain expression, confirming that they were constituents of the myeloma clone (Supplemental Figure 1, right column). The remaining 2 of 9 evaluable subjects did not exhibit CD19+ subpopulations. These observations indicate that minor $\mathrm{CD} 9^{+}$subpopulations of myeloma plasma cells are a 

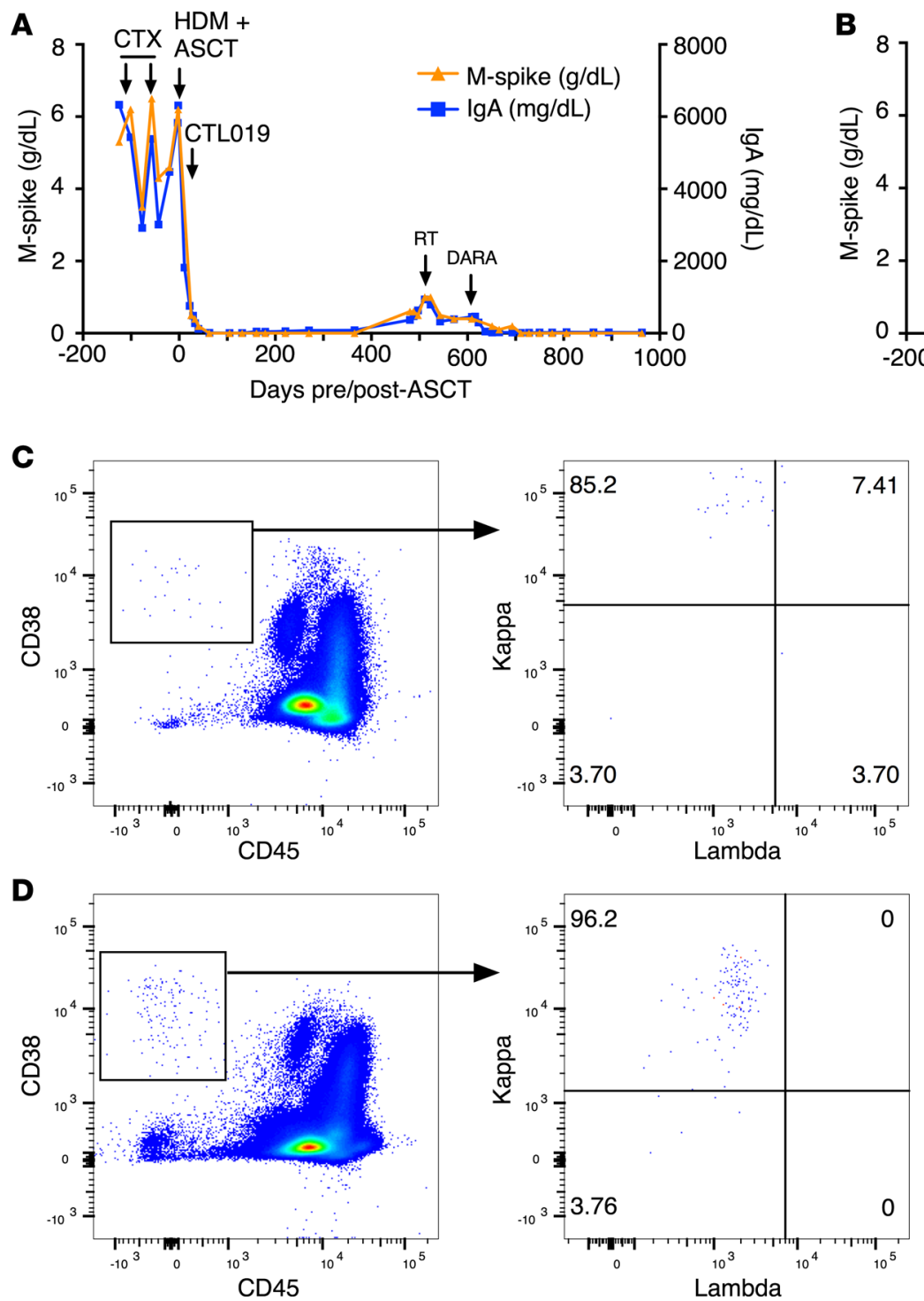
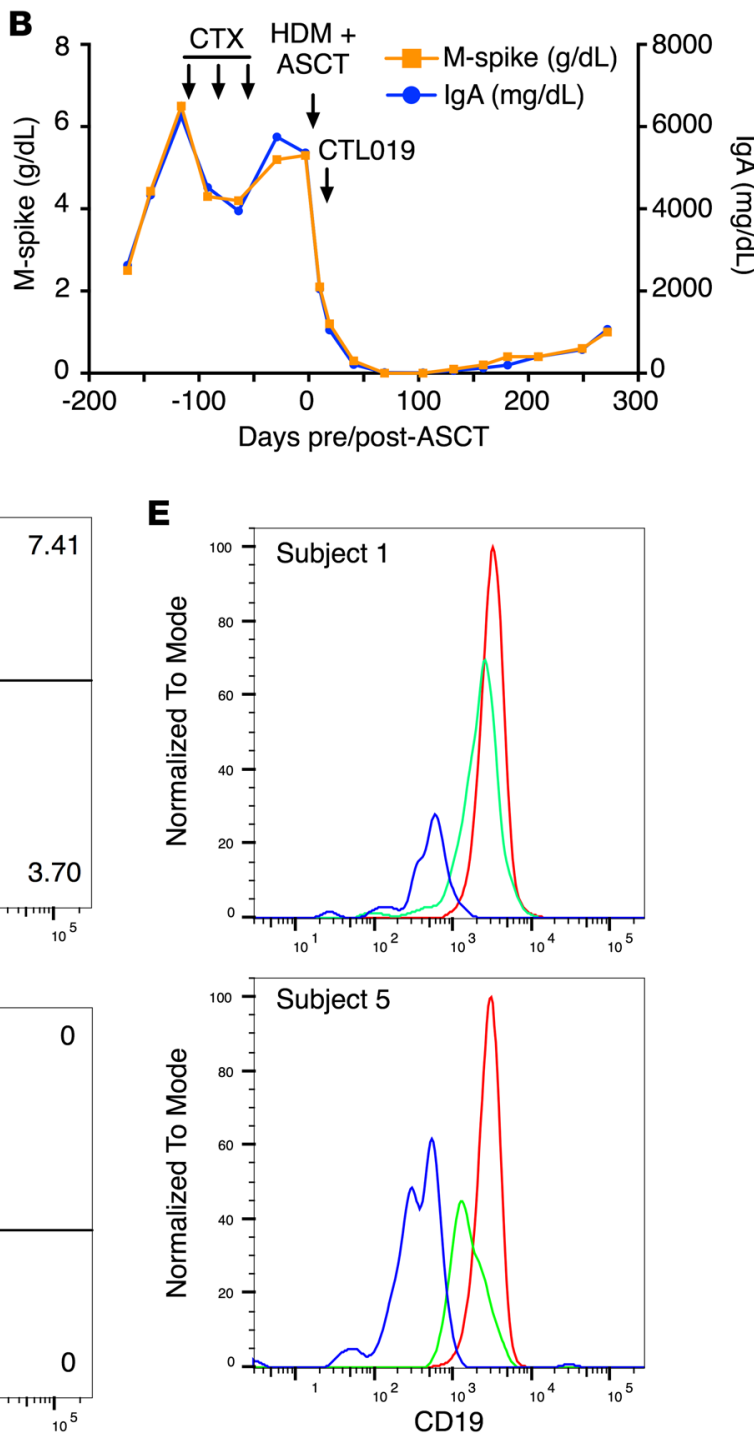

Figure 2. Clinical response and residual disease characterization in subjects $\mathbf{1}$ and $\mathbf{5}$. Trend in serum monoclonal protein concentration (M-spike) and total serum IgA in subjects 1 and 5 before and after ASCT + CTL019. (A) Subject 1. (B) Subject 5. CTX, 96-hour continuous-infusion cytotoxic chemotherapy; HDM, high-dose melphalan; RT, radiation therapy; DARA, daratumumab. (C and D) Flow cytometry to detect rare multiple myeloma plasma cells

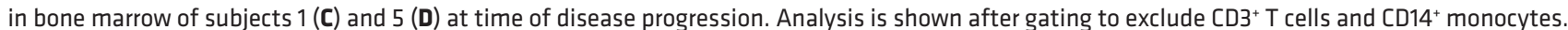
Rare CD $38^{+}$CD45 $5^{-}$plasma cells are gated (left) and shown to have kappa-restricted intracellular immunoglobulin light chain expression (right), consistent with a monoclonal population. (E) CD19 expression on rare multiple myeloma plasma cells (blue) is compared to CD19 on non-neoplastic plasma cells (CD38 ${ }^{\text {hi }} \mathrm{CD} 45^{+}$, green) and non-neoplastic B cells (CD19+CD20+, red) in bone marrow samples from subjects 1 (top) and 5 (bottom).

common feature of advanced multiple myeloma. However, there was no correlation between the frequency of this population and clinical outcome.

Anti-CD19 activity and in vivo persistence of CTL019. CTL019 cells were detected in peripheral blood of all subjects for a median of 43.5 days after infusion (range 14-156 days). Frequency of peripheral blood CD19+ B cells was generally low at time points when CTL019 cells were detectable and increased as CTL019 frequency decreased, indicating in vivo cytotoxicity by CTL019 against physiologic CD19+ cells (Supplemental Figure 2A). Consistent with the absence of clinically evident cytokine release syndrome, there were no significant elevations in IL-6 after CTL019 infusion except for subject 12, whose serum IL-6 rose but only to a modest degree compared with the much larger rises observed in subjects who have developed cytokine release syndrome in other studies with CTL019 (Supplemental Figure 3) (41, 42).

There was no significant correlation between the peak frequency or duration of CTL019 persistence in peripheral blood and clinical outcomes (Supplemental Figure 2, B and C). CTL019 cells were detectable 


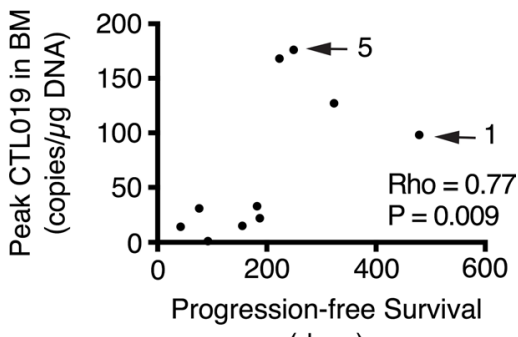

(days)
Figure 3. Correlation of progression-free survival versus peak CTL019 frequency in bone marrow (BM). Rho and $P$ values were determined using Spearman's correlations. Numbers refer to subjects 1 and 5.

in bone marrow in 9 of 10 subjects, and there was a significant correlation between the peak frequency of CTL019 cells in bone marrow and PFS (Spearman's rho $=0.77, P=0.009$; Figure 3).

Anti-Sox2 immune responses after CTL019. Expression of Sox2, a transcription factor that governs selfrenewal and pluripotency (43), has been linked to myeloma-propagating capability in myeloma cell lines $(44,45)$. Anti-Sox 2 immune responses may constrain the growth of monoclonal gammopathy of undetermined significance (MGUS) $(46,47)$, an indolent clonal plasma cell disorder that precedes multiple myeloma in most cases (48). Since the multiple myeloma in subjects 1 and 5 transitioned to an indolent clinical behavior after ASCT + CTL019, and since this indolent course continued after waning of in vivo CTL019 activity, we hypothesized that new anti-Sox 2 immune responses would be detectable and persist after CTL019 in these subjects.

We first examined anti-Sox 2 antibody responses before and after CTL019 by ELISA. Though all subjects had similarly low anti-Sox 2 antibody titers prior to ASCT, anti-Sox 2 titers rose specifically in subjects 1 and 5 , the subjects with the best clinical responses, at the 6- to 12-month post-ASCT time points (Figure 4A). Comparing the trend in anti-Sox 2 antibody responses over time in subjects 1 and 5 to the remaining subjects in a linear mixed-effects model, the rise in subjects 1 and 5 was significantly different $(P=$ 0.04). The induction of anti-Sox 2 antibodies in subject 1 was particularly strong, as an induction was still clearly apparent after serial dilution of the serum (Figure 4A). These anti-Sox 2 antibody responses were not likely due to general post-ASCT immune reconstitution alone since anti-tetanus antibody concentrations declined over the same time period in a subset of subjects analyzed, including subjects 1 and 5 (Figure 4A).

We also examined anti-Sox $2 \mathrm{~T}$ cell responses by stimulating subjects' peripheral blood mononuclear cells (PBMCs) with mixes of overlapping Sox2 peptides (mixes 1-2 and 3-4). Antigen-specific T cell proliferation was detected by CFSE dilution. Similar to the results with anti-Sox 2 antibody responses, subject 1 exhibited higher frequency of Sox2-reactive T cells and more sustained reactivity compared with the other subjects (Figure 4B). New post-ASCT reactivity in subject 1 was particularly distinctive against peptide mixes 3-4, against which pre-ASCT reactivity was low in all subjects; only subject 1 exhibited sustained postASCT reactivity against mixes 3-4 in both $\mathrm{CD} 4^{+}$and $\mathrm{CD} 8^{+}$subsets. At its peak in subject 1,6-9 months after ASCT, reactivity to mixes 3-4 was detected in $2 \%$ of all $\mathrm{T}$ cells (Figure $4 \mathrm{C}$ ). Further analysis revealed that this reactivity was localized to amino acids 194-219 of the Sox 2 protein sequence (Figure 4D). Detectable reactivity among $\mathrm{CD}^{+} \mathrm{T}$ cells against Sox2 peptide mixes 3-4 was sustained in subject 1 at time of isolated extramedullary disease progression, 494 days after ASCT (Figure 4B). Reactivity against control antigens (cytomegalovirus, Epstein-Barr virus, influenza [CEF]) in subject 1 remained below background at time points of peak anti-Sox 2 reactivity, indicating that anti-Sox 2 responses in subject 1 were not a by-product of nonspecific post-ASCT immune reconstitution (Figure 4C). Anti-Sox2 T cell responses were not observed in subject 5 despite the anti-Sox 2 antibody responses this subject exhibited (albeit at lower levels than subject 1).

The specific emergence of anti-Sox 2 immune responses after CTL019 in subjects with the most favorable clinical outcomes suggests that these immune responses were secondary to CTL019 activity. These responses could reflect epitope spreading due to coexpression of CD19 and Sox2 in myeloma-propagating cells targeted by CTL019.

In vitro myeloma-propagating capability of $C D 19^{-}$and BCMA-expressing multiple myeloma cells. Our observation that only 2 of 10 subjects benefitted from CTL019 could have been due to failure of CTL019 to eradicate its target in most subjects, perhaps due to inadequate cell dose and inadequate bone marrow engraftment. Alternatively, lack of clinical benefit could have been due to absence of CD19 expression on myeloma-propagating cells in many subjects. To better understand the heterogeneous effects of CTL019 on myeloma-propagating capability, we treated fresh bone marrow aspirate from multiple myeloma patients 
A

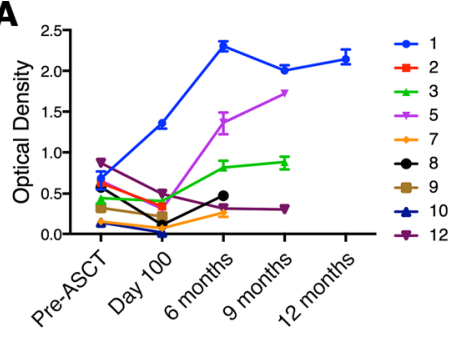

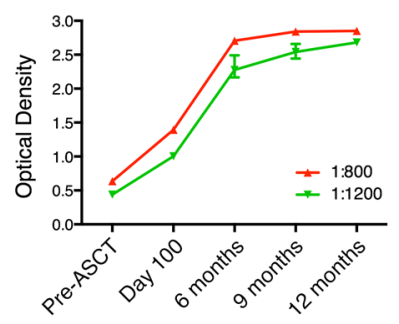

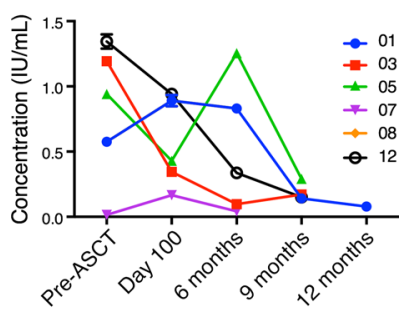

Sox2 Mixes3-4
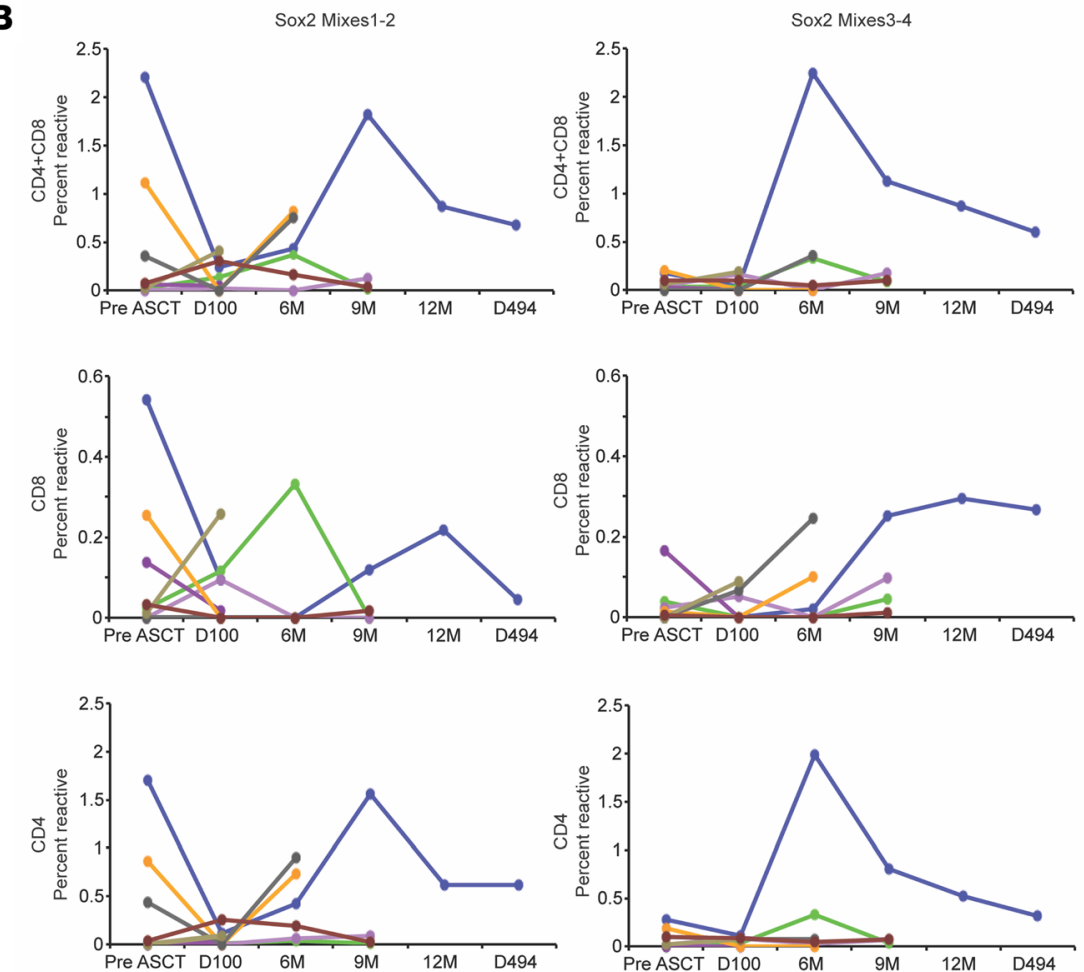

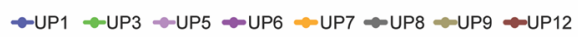

C
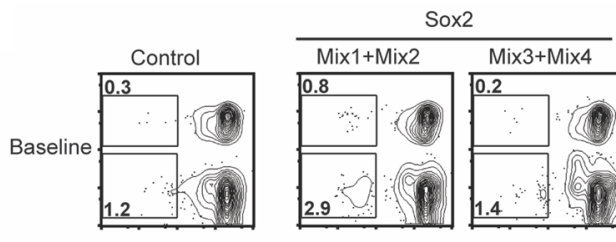

$6 \mathrm{M}$
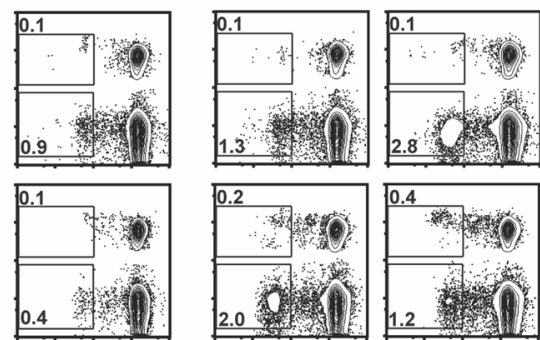
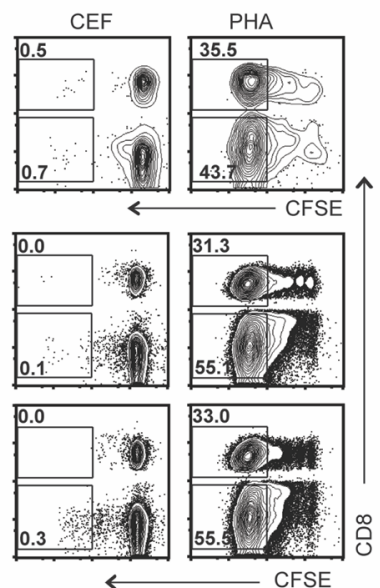
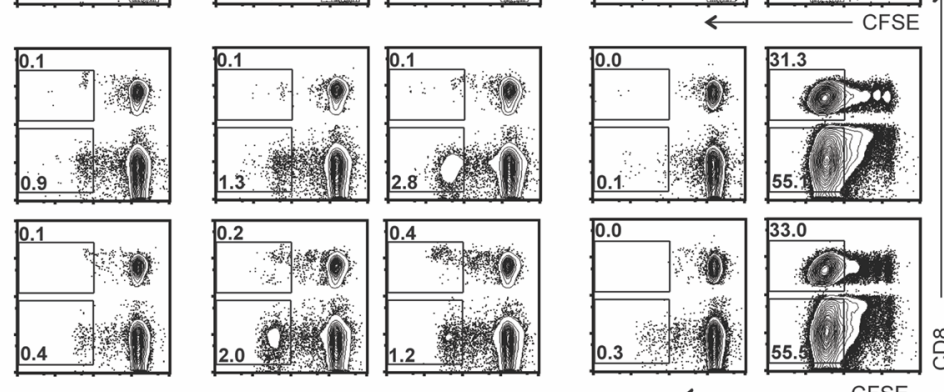

D
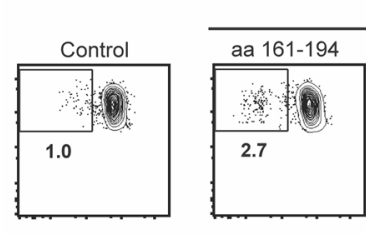

Sox2 Mix3

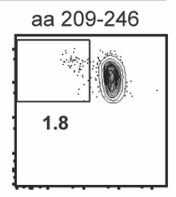

$\mathrm{PHA}$
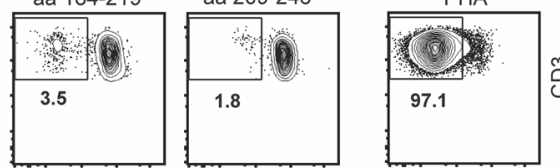

Figure 4. Anti-Sox2 immune responses after ASCT + CTL019.

(A) Left panel shows anti-Sox2 antibody concentrations before and after CTL019 + ASCT as measured by ELISA in serum diluted 1:400. Subject 6 was excluded from anti-Sox2 antibody analysis due lack of post-ASCT samples. Middle panel shows serum antiSox2 antibody concentrations in subject 1 after dilution of serum to $1: 800$ (red) and 1:1,200 (green) Right panel shows serum antitetanus toxoid antibody concentrations before and after CTL019 + ASCT; this analysis was restricted to subjects with samples available from 4 or more time points after ASCT. Data points indicate means, and error bars indicate range, of triplicate experiments after background subtraction. (B) Percentage of $T$ cells proliferating as assessed by CFSE dilution in response to Sox2 peptide mixes 1-2 (left) or 3-4 (right) from subjects' PBMCs collected before ASCT or at various time points after ASCT. Results are presented for all T cells (top row), CD8 ${ }^{+} \mathrm{T}$ cells (middle row), and $\mathrm{CD}^{+} \mathrm{T}$ cells (bottom row), after subtraction of percentages proliferating in unstimulated (vehicle-only) cultures. Subjects 2 and 10 were excluded from this analysis due to insufficient sample. (C) Subject 1 CFSE dilution in PBMCs collected prior to ASCT (top) and at 6 (middle) and 9 (bottom) months after ASCT in response to stimulation with Sox2-derived peptide mixes (mixes 1-2 or mixes $3-4$ ), negative control stimulation with vehicle only, CEF peptides (peptides derived from cytomegalovirus, Epstein-Barr virus, or influenza virus), or positive control stimulation with PHA. (D) Reactivity in subject 1 PBMCs obtained 12 months after ASCT against individual overlapping Sox2 peptides from mix 3 . 

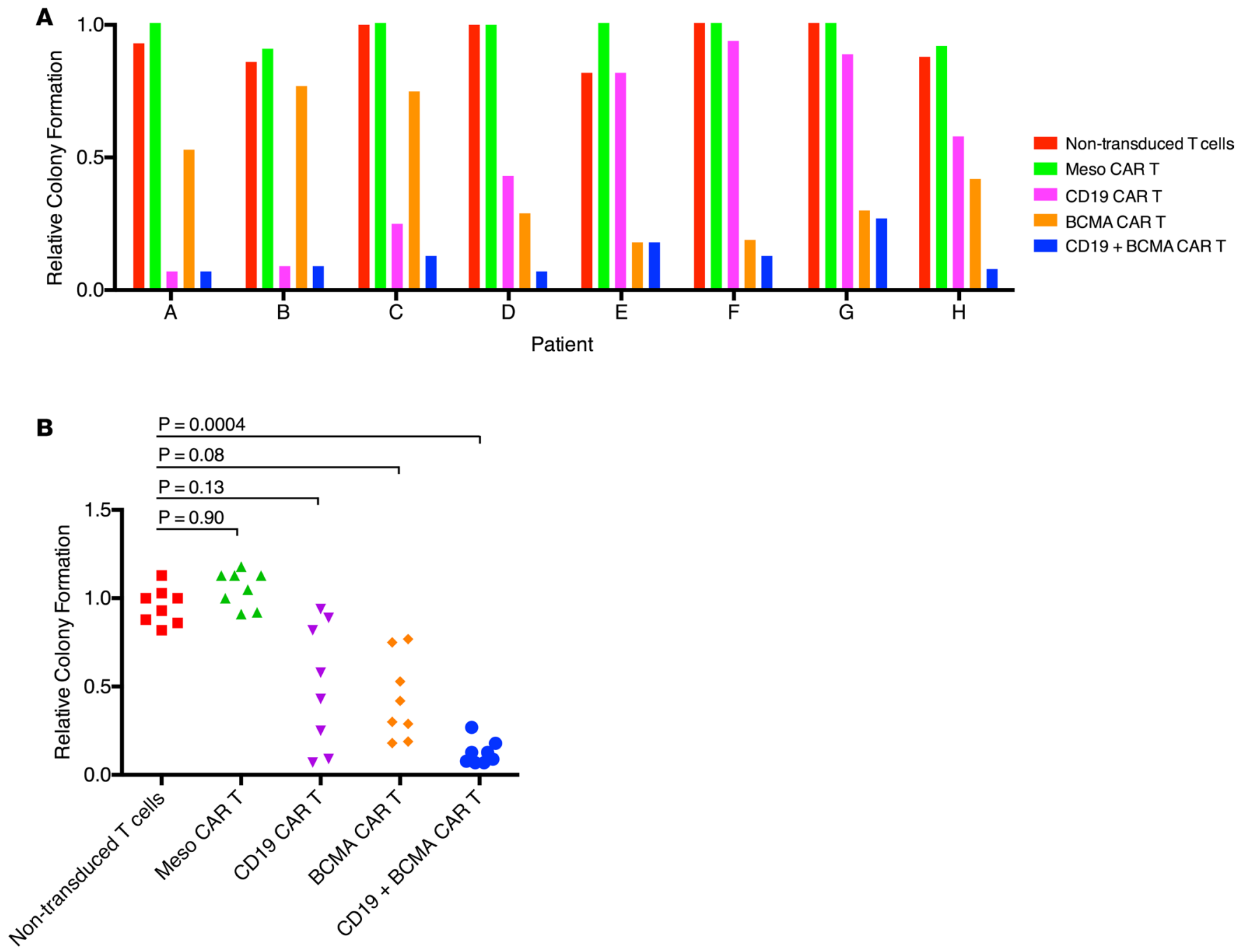

Figure 5. In vitro colony formation of bone marrow aspirate obtained from multiple myeloma patients not treated on our clinical trial following ex vivo incubation with CAR T cells against CD19 and/or BCMA, CAR T cells against mesothelin (meso CAR T), or nontransduced T cells. Results are normalized to untreated (no T cells) control and presented for each individual patient (A) and in aggregate by treatment condition (B). Groups were compared using Kruskal-Wallis test. $P$ values are reported from Dunn's test after Sidak's correction for multiple comparisons.

who were not enrolled on our study ex vivo with T cells transduced with either an anti-CD19 CAR (i.e., CTL019), a CAR against B cell maturation antigen (BCMA), or both. BCMA is a plasma cell antigen that is not expressed on most B cells; as we and others have reported, anti-BCMA CAR T cells have clinical activity in multiple myeloma (49-51). Since the CAR T cells in these experiments were derived from allogeneic healthy donors, we also treated aliquots with CAR T cells against an irrelevant target (mesothelin) and untransduced $\mathrm{T}$ cells to control for allo-immune effects. Following ex vivo treatment, bone marrow aspirates were depleted of CAR T cells by negative immunomagnetic selection and plated in methylcellulose to assess colony formation as an in vitro measure of myeloma-propagating capability (Figure 5). In all cases, as expected, untransduced $\mathrm{T}$ cells or anti-mesothelin $\mathrm{T}$ cells had no significant effect on colony formation compared with untreated controls. In contrast, both anti-CD19 and anti-BCMA CARs each individually depleted colony-formation capability, but they did so in different patients (Figure 5A). In 3 of 8 cases each, either anti-CD19 (patients A-C) or anti-BCMA CARs (patients E-G) were individually more effective; in the remaining 2 of 8 cases, both anti-CD19 and anti-BCMA CARs each partially depleted colony-formation capability to a similar degree (patients $\mathrm{D}$ and $\mathrm{H}$ ). In all cases, however, combined treatment with both anti-CD19 and anti-BCMA CARs resulted in significant decrease in colony formation compared with controls. Thus, analyzing results from all patients collectively (Figure 5B), only combined treatment with both anti-CD19 and anti-BCMA CARs resulted in a consistent, significant reduction in myeloma-propagating 
capability (multiplicity adjusted $P=0.004$ ). These results suggest that the surface immunophenotype of myeloma-propagating cells variably resembles B cells or plasma cells in different patients and that cotargeting both B cell and plasma cell antigens most reliably depletes myeloma-propagating capability.

\section{Discussion}

We conducted a clinical trial in which patients with advanced multiple myeloma received CTL019, an autologous anti-CD19 CAR T cell product, after high-dose melphalan and ASCT. We hypothesized that CTL019 would improve PFS after ASCT based on reports that minor CD19+ populations in otherwise CD19- myeloma harbor enhanced myeloma-propagating capability compared with the dominant CD19plasma cell population. We enrolled patients who had previously exhibited early progression after a prior ASCT and found that PFS after ASCT + CTL019 significantly exceeded prior PFS in 2 of 10 subjects. This prolonged PFS was not likely due to ASCT alone, as we did not observe any cases in our historical cohort where second ASCT alone resulted in better PFS than first-line ASCT. Moreover, our patient with the most prolonged PFS exhibited new anti-myeloma immunity, indolent clinical progression, and unusually durable response to subsequent therapy, suggesting that CTL019 favorably altered the clinical disease trajectory.

Our correlative data offer several possible reasons why only 2 of our 10 subjects exhibited clinical benefit from CTL019. First, CTL019 may not have effectively targeted CD19+ myeloma cells due to inadequate in vivo engraftment. Supporting this possibility, we observed a significant correlation between the peak frequency of CTL019 cells in bone marrow and PFS. The CTL019 dose used in this study was 10-fold lower than that used in most other studies of CTL019 $(41,42)$ due to concerns about the safety of combining CTL019 and ASCT. This low dose, along with the 12-day delay between lymphodepleting chemotherapy (i.e., the high-dose melphalan administered with ASCT) and CTL019 infusion, likely contributed to low peak engraftment and short in vivo persistence of CTL019 cells compared with what has been observed in other studies utilizing higher CTL019 doses and closer proximity of CTL019 infusion to chemotherapy. A second possible explanation for our heterogeneous responses is that myeloma-propagating cells may not be $\mathrm{CD}_{1}{ }^{+}$in all patients. Though we were not able to functionally assay myeloma-propagating capability in fresh samples from our clinical trial subjects, our analysis of bone marrow aspirates from a separate cohort of patients showed that ex vivo treatment with either CTL019 or anti-BCMA CAR T cells alone failed to reliably eliminate in vitro clonogenicity (Figure 5). We therefore suspect that surface immunophenotype is an imperfect predictor of myeloma-propagating potential; rather, the cell-surface immunophenotype of clinically important myeloma-propagating populations likely varies from patient to patient and may even change over time within individual patients during the long natural history of multiple myeloma. From a therapeutic perspective, together with evidence that less mature phenotypes become more frequent with successive lines of therapy $(8,52)$, our results provide rationale to broadly target both B cells and plasma cells to maximize the likelihood of eliminating all aspects of the myeloma clone, both the dominant plasma cell population and minor populations with less mature phenotypes. Indeed, we observed that ex vivo treatment of primary myeloma samples with both CTL019 and anti-BCMA CAR T cells significantly reduced in vitro clonogenicity in all cases examined (Figure 5).

The 2 subjects who exhibited prolonged PFS compared with prior ASCT both progressed with indolent, extramedullary plasmacytomas. Despite persistence of rare multiple myeloma plasma cells in bone marrow in both subjects, overt bone marrow relapse was not apparent. Isolated extramedullary progression (i.e., extramedullary progression without contemporaneous overt marrow progression) is unusual after standard myeloma therapy but is more common after allogeneic stem cell transplantation, often in association with chronic GVHD (53-56). A similar phenomenon has been observed in acute leukemia patients treated with allogeneic stem cell transplantation (57). Thus, isolated extramedullary relapse may indicate effective anti-myeloma immune surveillance of the bone marrow. Moreover, extramedullary progression in these subjects occurred long after CTL019 cells were no longer detectable in circulation or marrow. These findings suggest that CTL019 induced clinically active, secondary immune responses that persisted even after loss of CTL019 engraftment. These secondary immune responses may be a result of epitope spreading, as CTL019 cells killed minor CD19+ components of the myeloma clone. Epitope spreading has previously been observed in patients with solid tumors treated with anti-mesothelin CAR T cells (58). Epitope spreading could potentially be enhanced by combining CAR T cell therapies with other immunomodulatory approaches such as immune checkpoint blockade. Such manipulations may prolong responses after CAR T cell therapy in cases where CAR T cells do not persist in vivo indefinitely. 
Though we did not in this study undertake a broad search for targets of epitope spreading, we were specifically interested in anti-Sox 2 immune responses due to the potential mechanistic role of Sox 2 in a myeloma-propagating phenotype $(44,45)$. Sox 2 is a transcription factor that maintains capacity for selfrenewal and pluripotency in embryonic stem cells (43). In some human cancers, Sox2 defines a subset of cells with unique capacity to propagate malignancy (i.e., cancer stem cells) $(59,60)$. For example, Sox 2 confers capacity for long-lived latency in breast cancer cells, and immune surveillance against these latent Sox2-expressing cells prevents metastatic outbreak (61). Prior studies have implicated Sox2 in myeloma-propagating phenotypes in vitro $(44,45)$. Spontaneous anti-Sox 2 immune responses are associated with decreased risk of progression from MGUS to symptomatic multiple myeloma but are generally not observed in patients with active myeloma except after allogeneic (but not autologous) stem cell transplantation $(46,47,62)$. Though anti-Sox 2 immune responses themselves may or may not be directly clinically active, anti-Sox 2 immune responses may be a marker of immune recognition and active surveillance against myeloma-propagating cells; this surveillance may contribute to clinical disease quiescence typical of MGUS, and loss of this surveillance may allow the aggressive growth that characterizes active multiple myeloma. Others have observed induction of anti-Sox 2 immune responses after immunotherapy targeting the B cell antigen CD20 (63) that, like CD19, is typically absent on multiple myeloma plasma cells. Our finding that anti-Sox 2 immune responses emerged in subjects 1 and 5 as their multiple myeloma transitioned to a more indolent clinical course suggests that induction of immunity against myeloma-propagating cells is a promising therapeutic strategy. Moreover, our results suggest that Sox2 expression may be a more biologically relevant marker of myeloma-propagating cells than any particular cell-surface immunophenotype. Identification and monitoring of Sox2-expressing cells in patients may enable more specific targeting of myeloma-propagating cells with immunotherapies or other modalities.

We suspect that immunogenic cytotoxicity by CTL019 against CD19+, Sox2-expressing myelomapropagating cells led to the anti-Sox 2 immune responses we observed in subjects 1 and 5 . These antiSox2 responses were unlikely a result of ASCT alone since prior studies have shown that new anti-Sox2 immune responses after ASCT are rare (62). An alternative explanation is that anti-Sox2 responses were due to expansion of preexisting anti-Sox $2 \mathrm{~T}$ cells during the CAR T cell manufacturing process. Our results do not exclude this possibility, but we think this is unlikely based on the emergence in subject 1 of anti-Sox $2 \mathrm{~T}$ cells long after peak in vivo expansion of CTL019 and reactivity against multiple Sox2 peptides, including those to which reactivity was not detected in pre-ASCT samples (i.e., mixes 3-4).

Our study has several limitations. As a small pilot study, we could not control for the effects of high-dose melphalan on PFS, which would require randomization between ASCT alone and ASCT + CTL019. Our patient-specific comparisons to PFS after prior ASCT and our comparisons to a historical cohort therefore provide only preliminary evidence of clinical benefit from CTL019, and our conclusions are based on clinical and correlative findings in only 2 subjects with favorable outcome. Nonetheless, our results provide clear basis for future investigations. Specifically, our results provide rationale for clinical studies testing whether CTL019 can increase the durability of response to anti-BCMA CAR T cells, for which phase 1 studies have shown promising safety and initial responses but durable response in only a subset of patients $(50,51,64)$.

In summary, our results provide clinical evidence that targeting CD19+ myeloma-propagating cells can yield clinical benefit. Our results also provide a framework for understanding heterogeneity between patients in the immunophenotype of myeloma-propagating cells. Beyond multiple myeloma, our results provide clinical evidence in support of the cancer stem cell hypothesis (65) and the ability of potent immunotherapies targeted to minor disease-propagating populations to favorably alter the clinical trajectory of advanced cancers.

\section{Methods}

Clinical trial design. The clinical trial was conducted at the University of Pennsylvania. Subjects were selected from the investigators' clinical practices. Adults 18-70 years old with multiple myeloma that had progressed by IMWG criteria within 1 year of a prior administration of $200 \mathrm{mg} / \mathrm{m}^{2}$ melphalan and were medically fit for a second administration of high-dose melphalan were eligible. Study intervention consisted of melphalan at $140-200 \mathrm{mg} / \mathrm{m}^{2}$ followed 2 days later by infusion of $\geq 2 \times 10^{6} / \mathrm{kg} \mathrm{CD} 34^{+}$cells and $14-16$ days later by infusion of $1 \times 10^{7}$ to $5 \times 10^{7} \mathrm{CAR}$-expressing T cells. Standard supportive care was provided after high-dose melphalan including hematopoietic support with transfusions and filgrastim. Maintenance lenalidomide beginning at approximately day 100 after ASCT was permitted if subjects had received maintenance lenalidomide after prior ASCT. The primary endpoint of the study was safety and feasibility of CTL019 administration in 
this clinical setting. Sample size of 10 evaluable subjects was based on investigators' assessment of sample size required to evaluate safety and feasibility. Secondary objectives were to evaluate in vivo CAR T cell frequency and activity and PFS. Response assessment and PFS were evaluated according to IMWG criteria (38).

CTL019 production. CTL019 cells were manufactured from an autologous leukapheresis product as previously described (42). Autologous T cells were stimulated by paramagnetic polystyrene beads coated with anti-CD3 and anti-CD28 monoclonal antibodies and transduced with a lentiviral vector encoding an anti-CD19 single-chain variable fragment linked to 4-1BB and CD3-zeta signaling domains as previously described during an ex vivo expansion period of 9-10 days.

Multiple myeloma immunophenotyping. Bone marrow aspirates were analyzed by flow cytometry with the following antibody panel: CD38-BV421 (catalog 303525), CD14-BV510 (catalog 301842), CD56-BV605 (catalog 318333), CD3-BV650 (catalog 317324), CD19-BV785 (catalog 302239), Ig Lambda-Alexa Fluor 488 (intracellular) (catalog 316612), CD138-PerCP/Cy5.5 (catalog 352310), Ig Kappa-APC (intracellular) (catalog 316509), CD45-Alexa Fluor 700 (catalog 304023), CD20-APC/Cy7 (catalog 302313) from Biolegend; and BCMA-PE (catalog FAB193P) from R\&D Systems. Permeabilization and fixation reagents were obtained from Becton Dickinson. Data were acquired on a Becton Dickinson LSR Fortessa flow cytometer and analyzed using FlowJo (Becton Dickinson).

Monitoring of in vivo CTL019 frequency and activity. Peripheral blood and bone marrow samples were processed and evaluated for CTL019 by flow cytometry using an antibody specific for the anti-CD19 idiotype and qPCR for lentiviral vector sequences as previously described (42).

Anti-Sox2 antibody detection. Ninety-six-well plates were coated overnight with recombinant human Sox 2 protein (Abcam) at a final concentration of $1 \mu \mathrm{g} / \mathrm{ml}$ in PBS at $4^{\circ} \mathrm{C}$. Plates were washed with PBS containing $0.1 \%$ Tween 20 and then blocked with PBS containing 10\% FBS and $0.1 \%$ Tween 20 for 2.5 hours at room temperature. Serum was diluted 1:400, 1:800, or 1:1,200 in blocking buffer and plates were incubated for 1 hour at $37^{\circ} \mathrm{C}$. After washing, secondary antibodies bound to horseradish peroxidase (Southern Biotech) were diluted 1:8,000 in blocking buffer and incubated for 1 hour at $37^{\circ} \mathrm{C}$. After washing, detection reagent TMB substrate (Thermo Fisher Scientific) was added, and the detection reaction was terminated after 30 minutes at room temperature with $2 \mathrm{~N} \mathrm{HCl}$. Absorption was measured at $450 \mathrm{~nm}$ using an ELx800 plate reader (BioTek Instruments).

Anti-Sox 2 T cell detection. An overlapping SOX2 peptide library encompassing the entire SOX2 protein was generated as previously described (47) and peptides pooled into 4 mixes (mix 1 containing SOX2 amino acids 1-89, mix 2 containing amino acids 79-171, mix 3 containing amino acids 161-246, and mix 4 containing amino acids 236-321). PBMCs collected and frozen before ASCT and at various time points following ASCT + CTL019 were thawed together, rested for 1 hour at $37^{\circ} \mathrm{C}$ in the incubator, labeled with $0.5 \mu \mathrm{M}$ CFSE (Molecular Probes), and cultured with $1 \mu \mathrm{g} / \mathrm{ml}$ anti-CD28 and anti-CD49d antibodies (BD Biosciences) and DMSO (vehicle/control) or SOX2 peptide mixes (mixes 1-2 and mixes 3-4; $2.5 \mu \mathrm{g} / \mathrm{ml}$ per peptide). CEF peptide mix (Sigma-Aldrich; $2.5 \mu \mathrm{g} / \mathrm{ml}$ ) and phytohemagglutinin (PHA) were used as controls. Antigen-specific T cell proliferation (assessed by CFSE dilution) was analyzed at day 5 using flow cytometry with live/dead dye (Invitrogen) to exclude dead cells.

Ex vivo CAR T cell incubations and multiple myeloma colony formation assays. Bone marrow mononuclear cells were cocultured with or without $\mathrm{T}$ cells that were nontransduced or transduced with CAR constructs against mesothelin, CD19, and/or BCMA for 4-6 hours in RPMI 1640 containing 10\% FBS. Cells were then magnetically depleted of $\mathrm{CD}^{+}$cells (Miltenyi Biotec) and then plated in methylcellulose as previously described (66). Myeloma tumor colonies were quantified using an inverted microscope 14-21 days after plating.

Statistics. PFS2/PFS1 ratios were compared between the study and historical cohorts using Wilcoxon's rank-sum test. Spearman correlations were used to assess correlation between CTL019 frequency/persistence and PFS. Differences among groups in in vitro colony formation assays were evaluated using Dunn's test after Kruskal-Wallis test and after Sidak's correction for multiple comparisons. Statistical analyses were performed with Stata (StataCorp). A $P$ value $<0.05$ was considered significant.

Study approvals. The protocol was approved by the Institutional Review Board of the University of Pennsylvania, the US Food and Drug Administration, and the Recombinant DNA Advisory Committee, and was conducted under a FDA-approved Investigational New Drug Application. All patients gave informed consent in accordance with the Declaration of Helsinki. This study was registered at clinicaltrials.gov (identifier NCT02135406). 


\section{Author contributions}

ALG, EAS, WTH, SFL, JJM, MK, MPC, WHM, MVD, K Dhodapkar, DTV, BMW, ADC, ECA, SNC, RMY, BLL, MCM, MVM, and CHJ designed and interpreted experiments. All authors participated in acquisition and/or analysis of data. All authors participated in drafting and revision of the manuscript.

\section{Acknowledgments}

We gratefully acknowledge study subjects and their families for their participation in this clinical trial. We thank Jonathan Agudelo and Lee Dengel for clinical research support. We thank Alex Malykhin, Andrew Fesnak, and staff of the Penn Clinical Cell and Vaccine Production Facility cell manufacturing and product testing. We thank Jeffrey Finklestein, David Ambrose, Farzana Nazimuddin, Vanessa Gonzalez, and staff of the Penn Translational and Correlative Studies Laboratory for support with sample processing, correlative studies, and data management. This research was funded by Novartis, grants from the NIH (K12CA076931 and T32CA009615 to A.L. Garfall, K08CA166039 to M.V. Maus, R01CA174951 and K24CA198315 to W.H. Matsui), and the Conquer Cancer Foundation Career Development Awards to A.L. Garfall and M.V. Maus.

Address correspondence to: Alfred L. Garfall, Perelman Center for Advanced Medicine, 12-SPE, 3400 Civic Center Boulevard, Philadelphia, Pennsylvania 19104, USA. Phone: 215.662.7910; Email: alfred. garfall@uphs.upenn.edu.

MVD, K. Dhodapkar, and RD's present address is: Emory School of Medicine, Atlanta, Georgia, USA.

1. Rajkumar SV, Kumar S. Multiple myeloma: diagnosis and treatment. Mayo Clin Proc. 2016;91(1):101-119.

2. Hajek R, Okubote SA, Svachova H. Myeloma stem cell concepts, heterogeneity and plasticity of multiple myeloma. Br J Haematol. 2013;163(5):551-564.

3. Yaccoby S. Two states of myeloma stem cells. Clin Lymphoma Myeloma Leuk. 2018;18(1):38-43.

4. Tembhare PR, et al. Flow cytometric differentiation of abnormal and normal plasma cells in the bone marrow in patients with multiple myeloma and its precursor diseases. Leuk Res. 2014;38(3):371-376.

5. Chaidos A, et al. Clinical drug resistance linked to interconvertible phenotypic and functional states of tumor-propagating cells in multiple myeloma. Blood. 2013;121(2):318-328.

6. Rawstron AC, et al. Circulating plasma cells in multiple myeloma: characterization and correlation with disease stage. $\mathrm{Br} \mathrm{J} H a e^{-}$ matol. 1997;97(1):46-55.

7. Paiva B, et al. Detailed characterization of multiple myeloma circulating tumor cells shows unique phenotypic, cytogenetic, functional, and circadian distribution profile. Blood. 2013;122(22):3591-3598.

8. Paiva B, et al. Differentiation stage of myeloma plasma cells: biological and clinical significance. Leukemia. 2017;31(2):382-392

9. Bagg A, Becker P, Bezwoda W, van Rensburg L, Mendelow B. Circulating monotypic B-cells in multiple myeloma: association with lambda paraproteins. Br J Haematol. 1989;72(2):167-172.

10. Billadeau D, Ahmann G, Greipp P, Van Ness B. The bone marrow of multiple myeloma patients contains B cell populations at different stages of differentiation that are clonally related to the malignant plasma cell. J Exp Med. 1993;178(3):1023-1031.

11. Pilarski LM, Belch AR. Circulating monoclonal B cells expressing P glycoprotein may be a reservoir of multidrug-resistant disease in multiple myeloma. Blood. 1994;83(3):724-736.

12. Bergsagel PL, Masellis Smith A, Belch AR, Pilarski LM. The blood B-cells and bone marrow plasma cells in patients with multiple myeloma share identical IgH rearrangements. Curr Top Microbiol Immunol. 1995;194:17-24.

13. Bergsagel PL, Smith AM, Szczepek A, Mant MJ, Belch AR, Pilarski LM. In multiple myeloma, clonotypic B lymphocytes are detectable among CD19+ peripheral blood cells expressing CD38, CD56, and monotypic Ig light chain. Blood. 1995;85(2):436-447.

14. Chen BJ, Epstein J. Circulating clonal lymphocytes in myeloma constitute a minor subpopulation of B cells. Blood. 1996;87(5):1972-1976.

15. Szczepek AJ, Seeberger K, Wizniak J, Mant MJ, Belch AR, Pilarski LM. A high frequency of circulating B cells share clonotypic Ig heavy-chain VDJ rearrangements with autologous bone marrow plasma cells in multiple myeloma, as measured by single-cell and in situ reverse transcriptase-polymerase chain reaction. Blood. 1998;92(8):2844-2855.

16. Pilarski LM, Giannakopoulos NV, Szczepek AJ, Masellis AM, Mant MJ, Belch AR. In multiple myeloma, circulating hyperdiploid B cells have clonotypic immunoglobulin heavy chain rearrangements and may mediate spread of disease. Clin Cancer Res. 2000;6(2):585-596.

17. Pilarski LM, Hipperson G, Seeberger K, Pruski E, Coupland RW, Belch AR. Myeloma progenitors in the blood of patients with aggressive or minimal disease: engraftment and self-renewal of primary human myeloma in the bone marrow of NOD SCID mice. Blood. 2000;95(3):1056-1065.

18. Rasmussen T, Jensen L, Honoré L, Johnsen HE. Frequency and kinetics of polyclonal and clonal B cells in the peripheral blood of patients being treated for multiple myeloma. Blood. 2000;96(13):4357-4359.

19. Rasmussen T, Jensen L, Johnsen HE. Levels of circulating CD19+ cells in patients with multiple myeloma. Blood. 2000;95(12):4020-4021.

20. Pilarski LM, et al. Multiple myeloma includes phenotypically defined subsets of clonotypic CD20 $0^{+}$cells that persist during 
treatment with rituximab. Clin Med Oncol. 2008;2:275-287.

21. Rasmussen T, et al. Identification of translocation products but not K-RAS mutations in memory B cells from patients with multiple myeloma. Haematologica. 2010;95(10):1730-1737.

22. Yaccoby S, Epstein J. The proliferative potential of myeloma plasma cells manifest in the SCID-hu host. Blood. 1999;94(10):3576-3582.

23. Pilarski LM, Belch AR. Clonotypic myeloma cells able to xenograft myeloma to nonobese diabetic severe combined immunodeficient mice copurify with CD34 (+) hematopoietic progenitors. Clin Cancer Res. 2002;8(10):3198-3204.

24. Pilarski LM, et al. Leukemic B cells clonally identical to myeloma plasma cells are myelomagenic in NOD/SCID mice. Exp Hematol. 2002;30(3):221-228.

25. Matsui W, et al. Characterization of clonogenic multiple myeloma cells. Blood. 2004;103(6):2332-2336.

26. Peacock CD, et al. Hedgehog signaling maintains a tumor stem cell compartment in multiple myeloma. Proc Natl Acad Sci USA. 2007;104(10):4048-4053.

27. Boucher K, et al. Stemness of B-cell progenitors in multiple myeloma bone marrow. Clin Cancer Res. 2012;18(22):6155-6168.

28. Hosen N, et al. CD138-negative clonogenic cells are plasma cells but not B cells in some multiple myeloma patients. Leukemia. 2012;26(9):2135-2141.

29. Kim D, Park CY, Medeiros BC, Weissman IL. CD19-CD45 low/- CD38 high/CD138+ plasma cells enrich for human tumorigenic myeloma cells. Leukemia. 2012;26(12):2530-2537.

30. Rapoport AP, et al. Combination immunotherapy using adoptive T-cell transfer and tumor antigen vaccination on the basis of hTERT and survivin after ASCT for myeloma. Blood. 2011;117(3):788-797.

31. Rapoport AP, et al. Molecular remission of CML after autotransplantation followed by adoptive transfer of costimulated autologous T cells. Bone Marrow Transplant. 2004;33(1):53-60.

32. Rapoport AP, et al. Restoration of immunity in lymphopenic individuals with cancer by vaccination and adoptive T-cell transfer. Nat Med. 2005;11(11):1230-1237.

33. Stadtmauer EA, et al. Transfer of influenza vaccine-primed costimulated autologous $\mathrm{T}$ cells after stem cell transplantation for multiple myeloma leads to reconstitution of influenza immunity: results of a randomized clinical trial. Blood. 2011;117(1):63-71.

34. Rapoport AP, et al. NY-ESO-1-specific TCR-engineered T cells mediate sustained antigen-specific antitumor effects in myeloma. Nat Med. 2015;21(8):914-921.

35. Garfall AL, et al. Chimeric antigen receptor T cells against CD19 for multiple myeloma. N Engl J Med. 2015;373(11):1040-1047.

36. Holmberg L, et al. Gastrointestinal graft-versus-host disease in recipients of autologous hematopoietic stem cells: incidence, risk factors, and outcome. Biol Blood Marrow Transplant. 2006;12(2):226-234.

37. Rapoport AP, et al. Rapid immune recovery and graft-versus-host disease-like engraftment syndrome following adoptive transfer of costimulated autologous T cells. Clin Cancer Res. 2009;15(13):4499-4507.

38. Durie BG, et al. International uniform response criteria for multiple myeloma. Leukemia. 2006;20(9):1467-1473.

39. Michaelis LC, et al. Salvage second hematopoietic cell transplantation in myeloma. Biol Blood Marrow Transplant. 2013;19(5):760-766.

40. Cavo M, et al. Role of 18F-FDG PET/CT in the diagnosis and management of multiple myeloma and other plasma cell disorders: a consensus statement by the International Myeloma Working Group. Lancet Oncol. 2017;18(4):e206-e217.

41. Maude SL, et al. Chimeric antigen receptor T cells for sustained remissions in leukemia. N Engl J Med. 2014;371(16):1507-1517.

42. Porter DL, et al. Chimeric antigen receptor T cells persist and induce sustained remissions in relapsed refractory chronic lymphocytic leukemia. Sci Transl Med. 2015;7(303):303ra139.

43. Boyer LA, et al. Core transcriptional regulatory circuitry in human embryonic stem cells. Cell. 2005;122(6):947-956.

44. Tai YT, et al. Bruton tyrosine kinase inhibition is a novel therapeutic strategy targeting tumor in the bone marrow microenvironment in multiple myeloma. Blood. 2012;120(9):1877-1887.

45. Tanno T, et al. Growth differentiating factor 15 enhances the tumor-initiating and self-renewal potential of multiple myeloma cells. Blood. 2014;123(5):725-733.

46. Spisek R, et al. Frequent and specific immunity to the embryonal stem cell-associated antigen SOX2 in patients with monoclonal gammopathy. J Exp Med. 2007;204(4):831-840.

47. Dhodapkar MV, et al. Prospective analysis of antigen-specific immunity, stem-cell antigens, and immune checkpoints in monoclonal gammopathy. Blood. 2015;126(22):2475-2478.

48. Weiss BM, Abadie J, Verma P, Howard RS, Kuehl WM. A monoclonal gammopathy precedes multiple myeloma in most patients. Blood. 2009;113(22):5418-5422.

49. Ali SA, et al. T cells expressing an anti-B-cell maturation antigen chimeric antigen receptor cause remissions of multiple myeloma. Blood. 2016;128(13):1688-1700.

50. Cohen $\mathrm{AD}$, et al. B-cell maturation antigen (BCMA)-specific chimeric antigen receptor T cells (CART-BCMA) for multiple myeloma (MM): Initial safety and efficacy from a phase I study. Blood. 2016;128(22):1147.

51. Berdeja JG, et al. Durable clinical responses in heavily pretreated patients with relapsed/refractory multiple myeloma: Updated results from a multicenter study of bb2121 anti-Bcma CAR T cell therapy. Blood. 2017;130(Suppl 1):740 LP-740. http://www. bloodjournal.org/content/130/Suppl_1/740.abstract.

52. Leung-Hagesteijn C, et al. Xbp1s-negative tumor B cells and pre-plasmablasts mediate therapeutic proteasome inhibitor resistance in multiple myeloma. Cancer Cell. 2013;24(3):289-304.

53. Pérez-Simón JA, et al. Reduced-intensity conditioning allogeneic transplantation is associated with a high incidence of extramedullary relapses in multiple myeloma patients. Leukemia. 2006;20(3):542-545.

54. Zeiser R, Deschler B, Bertz H, Finke J, Engelhardt M. Extramedullary vs medullary relapse after autologous or allogeneic hematopoietic stem cell transplantation (HSCT) in multiple myeloma (MM) and its correlation to clinical outcome. Bone Marrow Transplant. 2004;34(12):1057-1065.

55. Minnema MC, et al. Extramedullary relapses after allogeneic non-myeloablative stem cell transplantation in multiple myeloma patients do not negatively affect treatment outcome. Bone Marrow Transplant. 2008;41(9):779-784.

56. Eefting M, et al. Donor T-cell responses and disease progression patterns of multiple myeloma. Bone Marrow Transplant. 
2017;52(12):1609-1615.

57. Shem-Tov N, et al. Isolated extramedullary relapse of acute leukemia after allogeneic stem cell transplantation: different kinetics and better prognosis than systemic relapse. Biol Blood Marrow Transplant. 2017;23(7):1087-1094.

58. Beatty GL, et al. Mesothelin-specific chimeric antigen receptor mRNA-engineered T cells induce anti-tumor activity in solid malignancies. Cancer Immunol Res. 2014;2(2):112-120.

59. Boumahdi S, et al. SOX2 controls tumour initiation and cancer stem-cell functions in squamous-cell carcinoma. Nature. 2014;511(7508):246-250.

60. Liu K, et al. The multiple roles for Sox2 in stem cell maintenance and tumorigenesis. Cell Signal. 2013;25(5):1264-1271.

61. Malladi S, et al. Metastatic latency and immune evasion through autocrine inhibition of WNT. Cell. 2016;165(1):45-60.

62. Kobold S, et al. Patients with multiple myeloma develop SOX2-specific autoantibodies after allogeneic stem cell transplantation. Clin Dev Immunol. 2011;2011:302145.

63. Lum LG, et al. Targeting CD138-/CD20+ clonogenic myeloma precursor cells decreases these cells and induces transferable antimyeloma immunity. Biol Blood Marrow Transplant. 2016;22(5):869-878.

64. Ali SA, et al. T cells expressing an anti-B-cell maturation antigen chimeric antigen receptor cause remissions of multiple myeloma. Blood. 2016;128(13):1688-1700.

65. Batlle E, Clevers H. Cancer stem cells revisited. Nat Med. 2017;23(10):1124-1134.

66. Matsui W, et al. Clonogenic multiple myeloma progenitors, stem cell properties, and drug resistance. Cancer Res. 2008;68(1):190-197. 